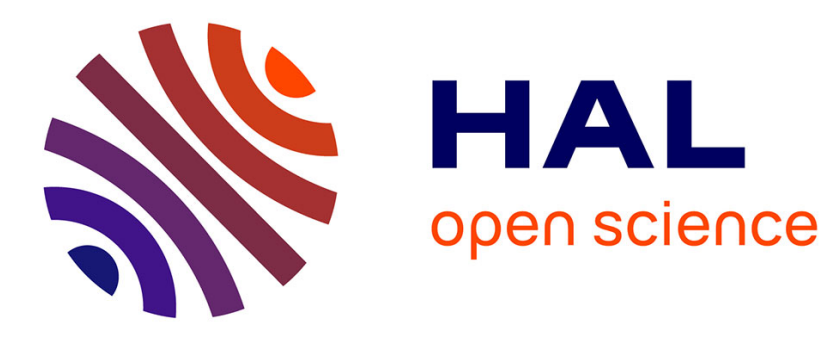

\title{
Direct Numerical Simulations of Two-Fluid Plasma Turbulence
}

\author{
A. Thyagaraja
}

\section{To cite this version:}

A. Thyagaraja. Direct Numerical Simulations of Two-Fluid Plasma Turbulence. Journal de Physique IV Proceedings, 1995, 05 (C6), pp.C6-105-C6-108. 10.1051/jp4:1995621 • jpa-00253989

\section{HAL Id: jpa-00253989 https://hal.science/jpa-00253989}

Submitted on 1 Jan 1995

HAL is a multi-disciplinary open access archive for the deposit and dissemination of scientific research documents, whether they are published or not. The documents may come from teaching and research institutions in France or abroad, or from public or private research centers.
L'archive ouverte pluridisciplinaire HAL, est destinée au dépôt et à la diffusion de documents scientifiques de niveau recherche, publiés ou non, émanant des établissements d'enseignement et de recherche français ou étrangers, des laboratoires publics ou privés. 


\title{
Direct Numerical Simulations of Two-Fluid Plasma Turbulence
}

\author{
A. Thyagaraja \\ UKAEA Government Division, Fusion, Culham, Abingdon, OX14 3DB, U.K. \\ (UKAEA/EURATOM Fusion Association)
}

\begin{abstract}
Electromagnetic turbulence thought to be responsible for anomalous transport in magnetic confinement devices such as tokamaks is very complicated, involving a multitude of physical processes, length and time-scales. It cannot be investigated by traditional linear theories any more than aerodynamic fluid turbulence. The relatively longer wavelength $\left(k_{\perp} \rho_{i} \ll 1\right)$, low frequency $\left(\omega \simeq \omega_{*} \ll \omega_{c i}\right)$ drift-type modes are, however, susceptible to a direct numerical solution approach pioneered in the case of fluid turbulence by Orszag and Patera. A substantial two-fluid nonlinear code called CUTIE has been developed at Culham in recent years to study the nonlinear saturation and transport consequences of electromagnetic drift wave turbulence in simplified tokamak geometry. This development and some results obtained using such a model are briefly described in this contribution.
\end{abstract}

\section{INTRODUCTION}

The CUTIE code simulates low frequency $\left(\omega \simeq \omega_{*} \ll \omega_{\text {ci }}\right)$, relatively long wavelength $\left(k_{\perp} \rho_{i} \ll\right.$ $1 ; m, n$ up to 20-30) drift-like fluctuations using a periodic cylinder $\left(r, \theta, \phi \equiv \frac{z}{R}\right)$ geometry and tokamak ordering (ie $\frac{a}{R} \ll 1, \frac{B_{\theta}}{B_{z}} \ll 1, \beta \ll 1$ )). Quasi-neutrality is assumed as well as suitable sources of particles, energy, current etc and transport co-efficients to take account of neoclassical 'parallel/perpendicular' effects. Standard nonlinear two-fluid+Maxwell equations (Refs. [1],[2]) are solved for the seven variables, $n_{e}, T_{e}, T_{i}, V_{\|}, \Phi, \Psi$ and $\Omega$ (parallel vorticity).

\section{EQUATIONS OF MOTION}

$$
\begin{aligned}
\frac{\partial n}{\partial t}+\nabla \cdot n \mathbf{V}_{M H D} & =S_{p}+\nabla \cdot D^{(n)} \nabla_{\perp} n \\
\frac{3}{2}\left[\frac{\partial p_{i}}{\partial t}+\nabla \cdot p_{i} \mathbf{V}_{M H D}\right] & =-p_{i} \nabla \cdot \mathbf{V}_{M H D}-\nabla \cdot \mathbf{q}_{i}+P_{\epsilon i}+S_{i} \\
\frac{3}{2}\left[\frac{\partial p_{e}}{\partial t}+\nabla \cdot p_{e} \mathbf{V}_{M H D}^{e}\right] & =-p_{e} \nabla \cdot \mathbf{V}_{M H D}^{e}-\nabla \cdot \mathbf{q}_{e}-P_{\epsilon i}+S_{e} \\
\frac{\partial \xi_{\|}}{\partial t}+\nabla \cdot \xi_{\|} \mathbf{V}_{M H D} & =-\nabla_{\|}\left(p_{i}+p_{e}\right)+\nabla \cdot D^{(\xi)} \nabla_{\perp} \xi_{\|}
\end{aligned}
$$




$$
\begin{aligned}
\frac{\partial \Psi}{\partial t}+\nabla \cdot\left(\Psi \mathbf{V}_{E}\right)+\frac{c \partial \Phi}{\partial z}+\frac{c \mathbf{b}}{e n} \cdot \nabla p_{e} & =\frac{c^{2} \eta}{4 \pi} \nabla_{\perp}^{2} \Psi+c E_{0 z} \\
\frac{\partial \Theta_{\|}}{\partial t}+\nabla \cdot \Theta_{\|} \mathbf{V}_{M H D}+n \mathbf{V}_{p i} \cdot \nabla\left(\frac{\Theta_{\|}}{n}\right) & =\frac{\mathbf{B}}{c} \cdot \nabla j_{\|}+\nabla \cdot D^{(\Theta)} \nabla_{\perp} \Theta_{\| !}
\end{aligned}
$$

The following auxiliary relations are used: $\xi_{\|} \equiv m_{i} n V_{\|}, \Theta_{\|} \equiv m_{i} n \frac{c}{B} \nabla_{\perp}^{2} \Phi ; \mathbf{V}_{E} \equiv \frac{c}{B} \mathbf{b} \times \nabla \Phi \simeq$ $\frac{c}{B} \hat{e}_{z} \times \nabla \Phi, \mathbf{V}_{p i} \equiv \frac{c}{e n B} \hat{e}_{z} \times \nabla p_{i} . \mathbf{V}_{M H D} \equiv \mathbf{V}_{E}+\mathbf{b} V_{\|}, \mathbf{V}_{M H D}^{e} \equiv \mathbf{V}_{M H D}-\mathbf{b} \frac{j_{\|}}{e n} ; j_{\|} \equiv-\frac{c}{4 \pi} \nabla_{\perp}^{2} \Psi$. In these equations, $S_{p}, S_{i}, S_{e}$ are specified sources. $E_{0 z}$ is the applied transformer field. $D^{(n)}, D^{(\xi)}, \frac{c^{2} \eta}{4 \pi}, D^{(\Theta)}$ are appropriate perpendicular diffusivities. In addition, $\chi_{\perp}^{(i)}, \chi_{\perp}^{(e)}, \chi_{\|}^{(i)}, \chi_{\|}^{(e)}$ must also be specified. Finally, $p_{e}=n T_{\epsilon}, p_{i}=n T_{i}$. Standard b.c's are applied.

\section{SOLUTION PROCEDURE AND RESULTS}

The mean equations take the form:

$$
\frac{\partial F}{\partial t}=\frac{1}{r} \frac{\partial}{\partial r}\left(r D \frac{\partial F}{\partial r}\right)+\sum(r, t)
$$

Finite-differencing yields a tri-diagonal matrix system which can be inverted by the well-known Gaussian elimination algorithm. The equations for the fluctuating variables also take an advectiondiffusion/parabolic form. Let $\tilde{F}(r, \theta, z, t)$ be the fluctuating quantity and advected by a velocity field $\mathbf{V}(r, \theta, z, t)$ which may be $\mathbf{V}$ or $\mathbf{V}_{M H D}$. All terms which are not advected or diffused are written as a source term $\sum^{(F)}(r, \theta, z, t)$. The equation of motion for $\tilde{F}$ is:

$$
\frac{\partial \tilde{F}}{\partial t}+\nabla \cdot \mathrm{V} \tilde{F}=\nabla \cdot D^{(F)} \nabla_{\perp} \tilde{F}+\tilde{\sum}^{(F)}
$$

where $\mathbf{V}$ is the full transporting velocity and $\tilde{\Sigma}^{(F)}$ is chosen to be consistent with $\langle\tilde{F}\rangle=0$ (ie $\tilde{F}$ has no $m=0$ component). In two-fluid theory, strong $\mathbf{E} \times \mathbf{B}$ flows are involved. Therefore the solution is carried out in poloidal planes by the standard Alternating Direction Implicit (ADI) scheme. Energy equations (involving large parallel transport) are solved by double Fourier transforms. Diffusion terms are treated implicitly for unconditional numerical stability.

We present typical results for conditions similar to COMPASS-D experiments. For details, Ref.[2] should be consulted. Two different spatial meshes, $(40 \times 20 \times 10$ and $25 \times 20 \times 15$ in $r, \theta, z$ directions respectively) were used, but the results do not appear to be significantly different. Calculations presented use the time-step, $2.5 \times 10^{-8} \mathrm{sec}$. Typical Alfvén speeds are $2.5 \times 10^{8} \mathrm{~cm} / \mathrm{sec}$, hence $V_{A} \Delta t / R \simeq 0.1$ for COMPASS-D $(a=18 \mathrm{~cm}, R=5.5 \mathrm{~cm})$. Electron thermal velocities $\left(T_{e} \simeq T_{i} \simeq\right.$ $1 \mathrm{keV}$ ) are of the order $10^{9} \mathrm{~cm} / \mathrm{sec}$. Hence $V_{\text {the }} \Delta t / R \simeq 0.5$. Initial $n_{e}$ is of order $5 \times 10^{13} / \mathrm{cc}$. All the simulations reported take $B_{0} \simeq 1$ Tesla and $q_{\text {edge }} \simeq 3$. The plasma current is held fixed at about $100 \mathrm{kA}$. We assume that $B_{0 \theta}(r, t)$ evolves according to Spitzer $\eta$. The turbulent fluctuations are subjected to an 'anomalous' diffusivity of the form, $\frac{C T_{0 \Omega}}{e B}\left\{\left|\frac{\delta j_{z}}{j_{z 0}}\right|+\lambda\left|\delta \Omega_{\|}\right|\right\}$; where $\lambda$ is a suitable normalising factor. This amounts to choosing a particular model of 'subgrid' turbulence. We take the self-induced mean poloidal $\mathbf{E} \times \mathbf{B}$ flow due to $\frac{d p_{0 i}}{d r}$ plus a fixed profile of toroidal flow of the form, $V_{0 \phi}(r)=V_{0}\left(1-\frac{r^{2}}{a^{2}}\right)$ with $V_{0}=5 \times 10^{6} \mathrm{~cm} / \mathrm{sec}$ and hold it constant through the calculation. 
Relatively strong particle and ion heating source terms were prescribed with ion temperatures essentially feed-back controlled. The electrons are heated ohmically and equilibrate with the ions. A simulation was carried out for 750 microseconds of 'real time'. The run took 35,000 seconds of Cray (ymp) cpu time. The plasma density rises linearly with $t$ from its initial central value of $5 \times 10^{13} / \mathrm{cc}$ to $4 \times 10^{15} / \mathrm{cc}$. This is of course rather unrealistic and due to the strong particle source. During this time the electron temperature rises monotonically from $0.1 \mathrm{keV}$ to $1.6 \mathrm{keV}$ and approaches saturation (ion temperature profile is fixed by feed-back). The loop voltage rises sharply to 2 volts in the first $100 \mu \mathrm{s}$ and then falls to a value of 0.3 volts (somewhat smaller than experiment but consistent with the electron temperature of $1.6 \mathrm{keV}$ ). The density fluctuations and the associated particle flux are essentially localised at the periphery (Fig 1 ). Contour plots of $\tilde{\Psi}, \tilde{\Phi}$ and $\Omega$ in a poloidal plane are shown in Figs.2-4. These correspond to the final epoch. The 'enstrophy' integral has been monitored as a function of time and shows saturation of the turbulence. The vorticity and current fluctuations show 'mesoscale' radial structures with radial correlations larger than grid-scales and Larmor radii. High densities (relatively smaller Alfvén speed) associated with strong particle sources appear to have a stabilizing effect on these nonlinearly saturated turbulent states. The effective confinement time is of the order of $1 \mathrm{~ms}$. This 'transient', driven, run with strong sources shows several characteristics of 'improved confinement' regimes in the presence of strong toroidal flows and sources.

\section{CONCLUSIONS}

This work gives an outline of the design philosophy and the methods employed in the CUTIE code to investigate the problem of turbulent plasma evolution under typical tokamak (COMPASSD) conditions. At this time, the challenge offered by this problem is truly formidable and the present attempt at its solution is one of many approaches[3] reported in the literature. The finer scales ('subgrid turbulence') are modelled phenomenologically. In spite of the relative simplicity of the physical model, the results presented are suggestive. Under the influence of sources, the system appears to become nonlinearly "self-organised" and evolves substantial (but saturated) fluctuations in the vorticity and current density. Quasi-coherent structures spontaneously appear in the simulation. In conclusion, CUTIE is a powerful computational tool capable of investigating tokamak turbulence and transport.

\section{Acknowledgements}

It is a pleasure to thank many useful and stimulating discussions with the following colleagues: Mike Bevir, Jack Connor, Steve Fielding, Jim Hastie, Chris Lashmore-Davies and Terry Martin.

\section{References}

[1] Hazeltine R.D. and Meiss J.D, Plasma Confinement(Addison-Wesley, New York, 1992) pp. $222-228$.

[2] Thyagaraja A., Direct numerical simulations of two-fluid plasma turbulence(UKAEA Govt. Division Fusion Report UKAEA FUS 303,Culham,1995)pp.1-29.

[3] Arter W., Reports on Progress in Physics, 58(1995) 1-10. 


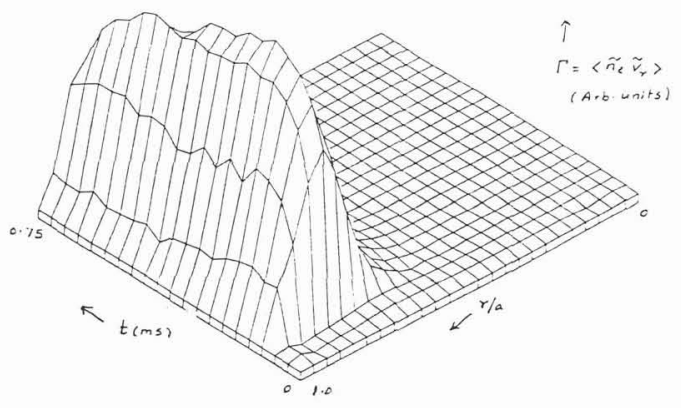

Figure 1: Calculated profile of the particle flux $\left\langle\delta n_{\mathrm{e}} \delta v_{r}\right\rangle(r, t)$. Note larger values occur near the edge $(r=a)$.

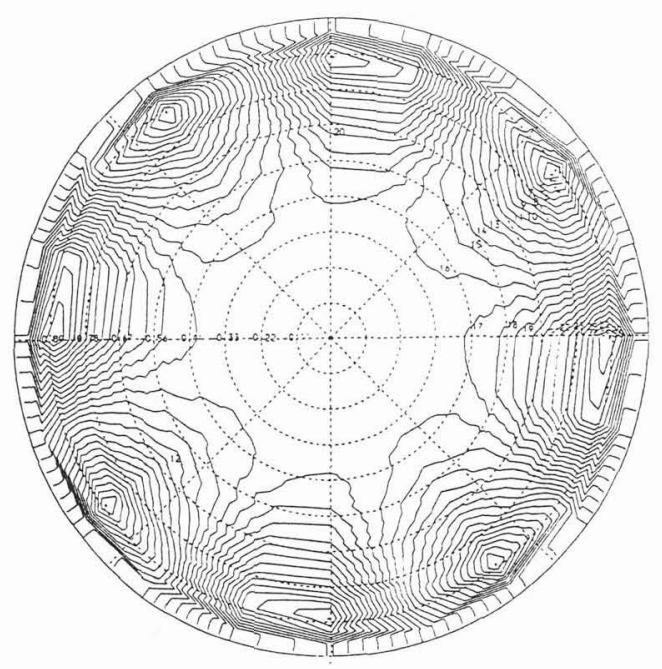

Figure 3: Contours of $\tilde{\Phi}$ in a poloidal plane at $0.75 \mathrm{msec}$.

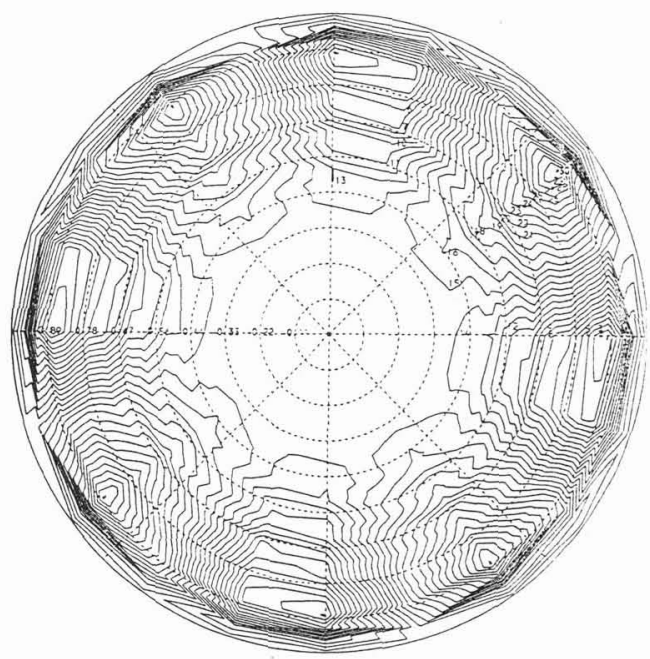

Figure 2: Contours of $\tilde{\Psi}$ in a poloidal plane at $0.75 \mathrm{msec}$.

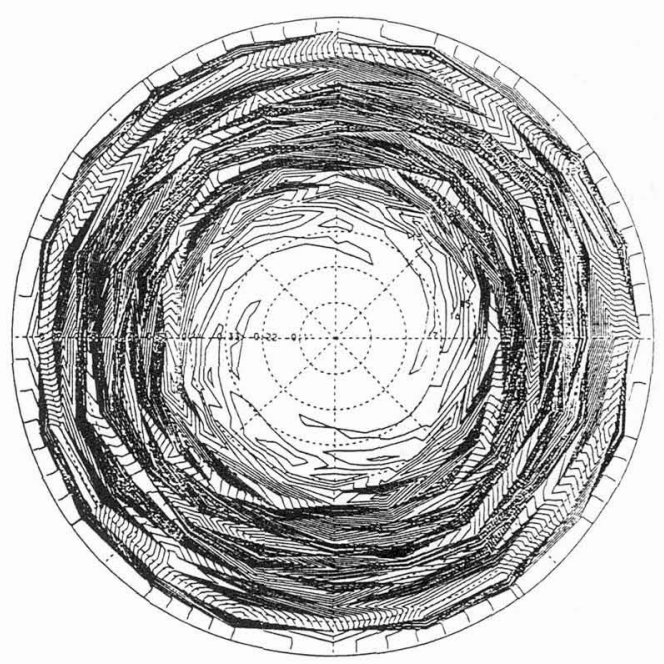

Figure 4: Contours of $\tilde{\Omega}_{\|}$in a poloidal plane at $0.75 \mathrm{msec}$. 